\title{
HAEFLIGER STRUCTURES AND LINEAR HOMOTOPY
}

BY

\author{
JAVIER BRACHO
}

\begin{abstract}
The notion of linear-homotopy into a classifying space is introduced and used to give a precise classification of Haefliger structures. Appendix on the product theorem for simplicial spaces and realizations of bisimplicial spaces.
\end{abstract}

Introduction. A concrete model for a classifying space is usually defined as the realization of a simplicial space. It is obtained by glueing continuous families of standard geometric simplexes, and thus, we have a nice coherent notion of linear simplex lying in it. This extra "linear structure" helps in trying to understand a classifying space geometrically and not only up to homotopy type. Thinking of points moving (' $p l$-ishly') along these simplexes, we get the notion of linearhomotopy, which, applied to Segal's model for the classifying space of a groupoid ([S] or (1.3)), gives a finer classification than Haefliger's classical one.

THEOREM B. For any topological groupoid $G$ and a locally compact space $X$, there is a one-to-one natural correspondence between linear-homotopy classes of maps from $X$ to $B G$, and $G$-structures on $X$.

This is the main result of the paper (observe (2.1)), so let us make it precise:

Given a simplicial space $A$, its geometric realization $|A|$ is obtained from $\prod A_{n} \times \Delta^{n}$ identifying with all the simplicial relations ([S] or (1.1)). Intuitively, think of $|A|$ as having two directions: a "continuous" one given by the topologies of the $A_{n}$ 's; and a "linear" one induced by the second factors. More precisely, consider the underlying simplicial set of $A: A^{\delta}$ (read $A$-discrete) obtained by forgetting the topologies of the $A_{n}$ 's. The set-theoretical identity gives us a map $\left|A^{\delta}\right| \rightarrow|A| \cdot\left|A^{\delta}\right|$ represents the "linear direction" of $|A|$.

Definition. A homotopy $H: X \times I \rightarrow|A|$ is a linear-homotopy if, for every $x \in X$, the path $H_{x}: I \rightarrow|A|$ factors through $\left|A^{\delta}\right| \rightarrow|A|$.

In terms of foliations: a linear-homotopy is one in which every point moves along its leaf (see §1(ii) for discussion and examples).

Received by the editors December 18, 1982.

1980 Mathematics Subject Classification. Primary 57R32, 54E99, 18G30; Secondary 55U40, 55R15.

'Part of this work is the author's Ph.D. Thesis (MIT, 1981), supervised by Professor D. M. Kan. Supported by CONACYT fellowship (17443), and UNAM. 
$G$-structures were defined by Haefliger in $[\mathbf{H}]$ (there, he called them $\Gamma$-structures but here $G$ will denote the groupoid). Haefliger's work opened broad perspectives to the theory of foliations through

Theorem A (HAefliger's Classification). There is a one-to-one natural correspondence between homotopy classes of maps from $X$ to $B G$, and homotopy classes of $G$-structures on $X$.

We venture a proof of Theorem A when $X$ is locally compact. It follows formally from Theorem B:

The correspondence is given by pulling back a universal $G$-structure on $B G$, thus, homotopic maps induce homotopic $G$-structures. On the other hand, suppose $f_{0}$ and $f_{1}: X \rightarrow B G$ induce $G$-structures $\xi_{0}$ and $\xi_{1}$ homotopic through a $G$-structure $\xi$ on $X \times I$. Use Theorem B to classify $\xi$ with a map $H: X \times I \rightarrow B G$, and then use it again to join the endmaps of $H$ to $f_{0}$ and to $f_{1}$ (through linear-homotopies- which are good enough).

In principle, Theorem B should enable us to work with Haefliger structures in terms more precise than "up to homotopy" (e.g., foliations up to isotopy). Unfortunately, we still do not know the invariants of linear-homotopy necessary to undertake such projects.

In the case where $G$ is a topological group, there is a simple application of Theorem B. Using the fact that (for a paracompact space $X$ ) homotopy of principal $G$-bundles (i.e., of $G$-structures) implies isomorphism, Theorems A and B yield that homotopy and linear-homotopy induce the same relation among maps from $X$ to $B G$, that is to say, every homotopy into $B G$ can be transformed into a linear-homotopy.

The paper is self-contained. $\$ 2$ can be considered as a precise proof of Theorem A. There, we develop the techniques necessary to prove the extra part of Theorem $B$ which is completed in $\$ 3$.

The Product Theorem (Theorem 2, Appendix) is essentially used twice within the text. Its particular application to the proof of Theorem B (part (ii) of Proposition 1, which is the "support" of the local compactness hypothesis) could have easily been left to the reader. But we preferred a general treatment in an Appendix to fill the void of a suitable reference in the literature.

Without the persistent encouragement and advice of Solomon Jekel, this work would have never achieved its present form. I thank him deeply. Also, I must acknowledge Daniel Kan's impressive introduction to the subject.

1. Definitions and examples. The definitions in (i) are well known [S]; we rush through them only to precise notation. In (ii) we discuss a little further the notion of linear-homotopy and detail the relevant examples. Haefliger structures are treated in (iii).

(i) Simplicial spaces, topological categories and groupoids. Let $\mathbf{n}$ denote the category naturally associated to the ordered set $\{0,1, \ldots, n\}$. Analogously, $\mathbf{N}$ has object set $\{0,1, \ldots\}$. 
(1.1) A simplicial space $A$ is a collection $\left\{A_{n}\right\}$ of spaces together with face maps $\mathbf{f}^{*}$ : $A_{m} \rightarrow A_{n}$ for every functor $\mathbf{f}: \mathbf{n} \rightarrow \mathbf{m}$. Its realization is the space $|A|=\prod_{n} A_{n} \times \Delta^{n} / \sim$, where $\left(a, \mathbf{f}_{*}(t)\right) \sim\left(\mathbf{f}^{*}(a), t\right)$ for every $\mathbf{f}: \mathbf{n} \rightarrow \mathbf{m}, a \in A_{m}$ and $t \in \Delta^{n}$.

Given a point $x \in|A|$, there is a unique nondegenerate simplex of $A, \operatorname{car}(x)$ (read carrier of $x)$, such that $\left(\operatorname{car}(x), t_{x}\right)$ represents $x, t_{x}$ is an interior point of $\Delta^{n}$, and $n=\operatorname{dim}(\operatorname{car}(x))$.

(1.2) Given a topological category (top-cat) $C, \mathrm{Ob}(C)(\operatorname{Mor}(C))$ denotes its object (morphism) space; $S \& T: \operatorname{Mor}(C) \rightarrow \mathrm{Ob}(C)$ denote the source and target maps. If every morphism $c \in \operatorname{Mor}(C)$ has an inverse $c^{-1}$, and if the involution $c \mapsto c^{-1}$ is continuous, $C$ is called a groupoid. $G$ will denote a groupoid throughout this work.

$X$ will denote a space, or the groupoid naturally associated to it: $\operatorname{Ob}(X)$ and $\operatorname{Mor}(X)$ are copies of $X$ and all structural maps are the identity map of $X$ ( $X$ as a groupoid is the initial object among top-cats having object space $X$ ). The context will always clarify the meaning of $X$ (although that, in a categorical sense, the difference is superfluous).

The nerve of a top-cat $C$ is the simplicial space $N C$ having $(N C)_{n}=$ Functors $\{\mathbf{n}$ $\rightarrow C$ \} with the subspace topology from $\operatorname{Mor}(C)^{\operatorname{Mor}(\mathbf{n})}$. And the realization of $C$ is $|C|=|N C|$.

Observe that if $x \in|C|$, then $|\operatorname{car}(x)|\left(t_{x}\right)=x$.

The explicit model for the classifying space of a groupoid that we use is Segal's generalization of Milnor's join construction [S]:

(1.3) Given a topological category $C$, let $C_{\mathbf{N}}$ denote the subtop-cat of $\mathbf{N} \times C$ obtained by deleting all nonidentity morphisms whose first component is an identity. Then define $B C=\left|C_{\mathbf{N}}\right|$.

(ii) Linear homotopy. $X^{\delta}$ (read $X$-discrete) denotes the space with the same pointset as $X$ but with the discrete topology. Thus, for a simplicial space $A$, we have $A^{\delta}=\left\{A_{n}^{\delta}\right\}$ and there is an obvious simplicial map $A^{\delta} \rightarrow A$. The analogous notation applies to top-cats.

Let $A$ be a simplicial space (or a top-cat). Consider a homotopy $H: X \times I \rightarrow|A|$. We always have a set theoretical commutative diagram:

$$
\begin{array}{ccc}
X^{\delta} \times I & \stackrel{H^{\prime}}{\rightarrow} & \left|A^{\delta}\right| \\
\downarrow & & \downarrow \\
X \times I & \stackrel{H}{\rightarrow} & |A|
\end{array}
$$

$H$ is a linear-homotopy if and only if $H^{l}$ is continuous.

Linear-homotopy is a natural equivalence relation to consider among maps into "classifying spaces". Here are some examples.

For a discrete simplicial space, linear-homotopy is homotopy in the usual sense.

On the other extreme, a linear-homotopy into a space $Y=|Y|$ is constant (as a homotopy). Thus, linear-homotopy is equality among spaces.

(1.5) Natural transformations induce linear-homotopies. This observation is due to Segal [S] (where we borrowed the term from). Let $T: C \times \mathbf{1} \rightarrow D$ be a (continuous) 
natural transformation $-C$ and $D$ are topological categories. The Product Theorem (Theorem 2, Appendix) gives us a homotopy $|T|:|C| \times I \rightarrow|D|$. But we also have a map $\left|T^{\delta}\right|:\left|C^{\delta}\right| \times I \rightarrow\left|D^{\delta}\right|$ through which $|T|^{l}$ clearly factors (see (1.4)). Whence, $|T|$ is, in fact, a linear-homotopy.

Finally, let us consider Haefliger's groupoid $\Gamma[\mathbf{H}] . B \Gamma$ is the "classifying space for foliations", and it is well known that $B \Gamma^{\delta}$ is the leaf of the universal foliation on $B \Gamma$. Thus, linear-homotopy is precisely "moving along the leafs". In this context, Theorem $\mathrm{B}$ is intuitively clear: the foliation induced by a map $f: X \rightarrow B \Gamma$ is not changed at all if we let $X$ move along the universal foliation.

(iii) Haefliger structures. The following definition is easily seen to correspond exactly to the original one of Haefliger [H].

First, we associate a groupoid to an open cover:

(1.6) Given $U=\left\{U_{\alpha}\right\}_{\alpha \in \Sigma}$, an open cover of $X$, denote by $\underline{\Sigma}$ the discrete groupoid with $\operatorname{Ob}(\underline{\Sigma})=\Sigma$ and $\operatorname{Mor}(\underline{\Sigma})=\Sigma \times \Sigma$ with the obvious structural maps. Then define $X_{U}$ to be the full subgroupoid of $X \times \underline{\Sigma}$ spanned by the subspace of objects: $\left\{(x, \alpha) \mid x \in U_{\alpha}\right\}$.

(1.7) Definition. A $G$-structure on $X$ is an equivalence class of $G$-cocycles on $X$; where a $G$-cocycle on $X$ is a pair $(U, F)$ with $U$ an open cover of $X$ and $F: X_{U} \rightarrow G$ a functor; and where two $G$-cocycles $\left(U_{0}, F_{0}\right)$ and $\left(U_{1}, F_{1}\right)$ on $X$ are equivalent if there exists a functor $F: X_{U_{0} \amalg U_{1}} \rightarrow G$ restricting (in the obvious sense) to $F_{0}$ and $F_{1}$. A $G$-structure on $X$ is numerable if it is represented by a $G$-cocycle $(U, F)$ with $U$ admitting a subordinate partition of unity.

Examples of $G$-structures form a wide spectrum: maps (when $G$ is a space); principal bundles (when $G$ is a group); foliations (for Haefliger's $\Gamma$ ); manifold or geometric structures (0-dimensional foliations for obvious choices of $\Gamma$ ), and vector bundle morphisms are some of them. For more discussion and examples see [H or B].

2. Proof of Theorem A. Partitions of unity play an important role in the classic proof of Theorem A. For expository reasons, we did not mention them in the Introduction. But it is due time to correct this omission:

(2.1) RemarK. In Theorems A and B, " $G$-structures" should read "numerable $G$-structures". Furthermore, in Theorem A, homotopies between numerable $G$-structures are understood to be numerable.

Here, instead of working with partitions of unity in the usual way, we work with (the closely related concept of) maps into the infinite simplex $\Delta^{\infty}(=|\mathbf{N}|$, as definition). So we have

(2.2) Definition. Given a map $p: X \rightarrow \Delta^{\infty}$, let $X_{p}$ be the full subtop-cat of $X \times \mathbf{N}$ spanned by the subspace of objects $\{(x, i) \mid i \in \operatorname{Image}(\operatorname{car}(p(x)))\}$. A numerated $G$-cocycle on $X$ is a pair $(p, F)$ where $p: X \rightarrow \Delta^{\infty}$ is a map and $F: X_{p} \rightarrow G$ is a functor. Denote by $\operatorname{NumCo}(X, G)$ the set of such.

(2.3) RemarK. Numerable $G$-structures on $X$ can be considered in a natural way as equivalence classes of numerated $G$-cocycles on $X$ under the obvious equivalence relation (see, e.g., (2.5)). 
This is so because a numerated $G$-cocycle on $X$ is essentially a $G$-cocycle together with a fixed subordinate partition of unity. (Compare (2.2) with (1.6) and (1.7), keeping in mind the natural open cover of $\Delta^{\infty}$ and that $G$ is a groupoid.) (Details can be found in $[\mathbf{B}]$ or in the author's Thesis.)

Matching the precision gained by (2.3), we have

Proposition 1. For a locally compact space $X$, and any groupoid $G$, there is a natural bijection

$$
\operatorname{Maps}(X, B G) \leftrightarrow \operatorname{NumCo}(X, G) .
$$

Proof. (i) Let $f: X \rightarrow B G$ be a given map. Recall that $B G=\left|G_{\mathrm{N}}\right|$ and that $G_{\mathbf{N}} \subset \mathbf{N} \times G$, (1.3). Let $\pi_{1}$ be the projection functor from $G_{\mathbf{N}}$ to $\mathbf{N}$. Then put $p=\left|\pi_{1}\right| \cdot f$.

To define $F: X_{p} \rightarrow G$, let $X_{f}$ be the subtop-cat of $X \times G_{\mathrm{N}}$ consisting of objects (and morphisms) $(x, g)$ such that $g \in \operatorname{Image}(\operatorname{car}(f(x))) . X_{f}$ is indeed a category for, by the definition of $G_{\mathbf{N}}$, the image of any simplex $\left(\mathbf{n} \rightarrow G_{N}\right)$ is already a subcategory of $G_{\mathbf{N}}$.

(2.4) Claim. The restriction to $X_{f}$ of the functor $\operatorname{id}_{X} \times \pi_{1}: X \times G_{\mathbf{N}} \rightarrow X \times \mathbf{N}$ is an isomorphism onto $X_{p}$.

Since $\pi_{1}$ takes car $(f(x))$ isomorphically onto car $(p(x))$ for every $x \in X$, then it is clear that $\left(\mathrm{id}_{X} \times \pi_{1}\right) \mid X_{f}$ is a bijection onto $X_{p}$. That it is, moreover, an isomorphism of top-cats follows from the fact that, since $\mathbf{N}$ is discrete, $\mathrm{id}_{X} \times \pi_{1}$ is open.

Observe that $X_{f}$ comes equipped with a functor $\tilde{f}: X_{f} \rightarrow G_{\mathrm{N}}$, so that we define $F$ as the composite

$$
X_{p} \rightarrow X_{f} \stackrel{\tilde{f}}{\rightarrow} G_{\mathbf{N}} \stackrel{\pi_{2}}{\rightarrow} G
$$

This gives the correspondence $\operatorname{Maps}(X, B G) \rightarrow \operatorname{NumCo}(X, G)$.

(ii) Take $(p, F) \in \operatorname{NumCo}(X, G)$. Let $\tilde{p}: X_{p} \rightarrow \mathbf{N}$ be the projection functor. Observe that $(\tilde{p}, F): X_{p} \rightarrow \mathbf{N} \times G$ factors through the inclusion $G_{\mathbf{N}} \hookrightarrow \mathbf{N} \times G$; so that we get a functor $\bar{F}: X_{p} \rightarrow G_{\mathbf{N}}$.

If $X$ is locally compact then $|X \times \mathbf{N}|=X \times \Delta^{\infty}$ (Theorem 2, Appendix), and then the graph of $p$ defines a section $s_{p}: X \rightarrow\left|X_{p}\right|$. This gives a map

$$
|\bar{F}| \cdot s_{p}: X \rightarrow B G \text {. }
$$

The rest of the proof is straightforward.

Proposition 2. Let $X$ be locally compact, and $G$ a groupoid. Let $f_{0}, f_{1}: X \rightarrow B G$ and $\left(p_{0}, F_{0}\right),\left(p_{1}, F_{1}\right) \in \operatorname{Num} \operatorname{Co}(X, G)$ correspond, respectively, to each other by means of Proposition 1, then

$$
\left(p_{0}, F_{0}\right) \sim\left(p_{1}, F_{1}\right) \Rightarrow f_{0} \text { is linear-homotopic to } f_{1} \text {. }
$$

Proof. Let $h_{0}, h_{1}: \mathbf{N} \rightarrow \mathbf{N}$ be the functors $h_{0}(n)=2 n, h_{1}(n)=2 n+1$. Let $p$ : $X \rightarrow \Delta^{\infty}$ be the map defined by

$$
p=\frac{1}{2}\left(\left|h_{0}\right| \cdot p_{0}\right)+\frac{1}{2}\left(\left|h_{1}\right| \cdot p_{1}\right)
$$


and let $i_{k}: X_{p_{k}} \rightarrow X_{p}, k=0,1$, be the obvious inclusions (restrict $\operatorname{id}_{X} \times h_{k}$ ). We clearly have

(2.5) $\left(p_{0}, F_{0}\right) \sim\left(p_{1}, F_{1}\right)$ iff there exists $F: X_{p} \rightarrow G$ such that $F_{0}=F \cdot i_{0}$ and $F_{1}=F \cdot i_{1}$.

Recalling part (ii) of Proposition 1, consider the diagram

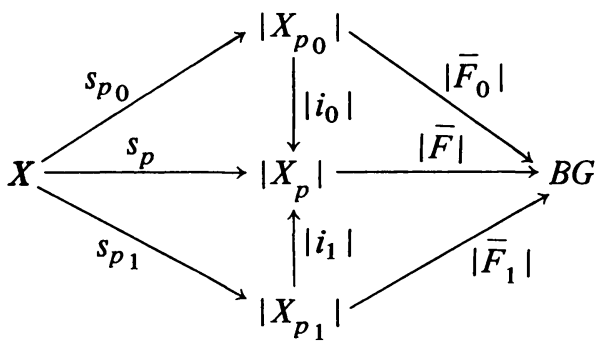

where $f_{0}$ and $f_{1}$ appear as the top and bottom row compositions respectively. We see that the diagram commutes up to linear-homotopy:

Clearly, the left-hand side triangles are linear-homotopy commutative (all the maps except the $F$ 's are linear-homotopy equivalences). On the other hand, the natural transformations from $\bar{F}_{k}$ to $\bar{F} \circ i_{k}$ defined by

$$
(x, n) \mapsto\left(n \rightarrow h_{k}(n), \operatorname{id}\left(F_{k}(x, n)\right)\right)
$$

for $(x, n) \in \mathrm{Ob}\left(X_{p_{k}}\right)$ and $k=0,1$, produce via (1.5) the required linear-homotopies for the right-hand side triangles.

At this point, the proof of Theorem A given in the Introduction works with two comments on it: the first assertion is clearly valid (reconsider part (i) of Proposition 1 with $\mathrm{id}_{B G}$ ); as for the applications of Theorem B, observe that Propositions 1 and 2 are (respectively) sufficient.

3. Proof of Theorem B. There only remains to prove the following

Proposition 3. With notation as in Proposition 2, we have

$$
f_{0} \text { is linear-homotopic to } f_{1} \Rightarrow\left(p_{0}, F_{0}\right) \sim\left(p_{1}, F_{1}\right) \text {. }
$$

Proof. Let $p: X \rightarrow \Delta^{\infty}$ be as in Proposition 2. From (2.5) it clearly suffices to fill the following diagram:

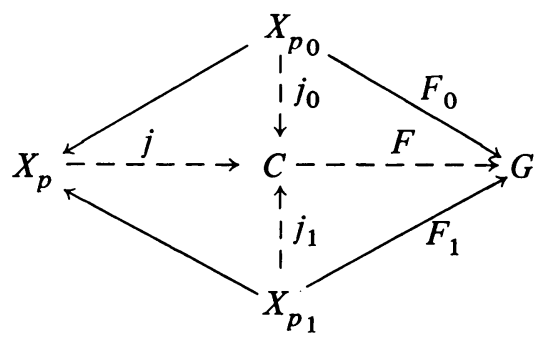


Let $H: X \times I \rightarrow B G$ be a linear-homotopy from $f_{0}$ to $f_{1}$.

(i) Construction of $C$. (a) Let $A$ be the subsimplicial-space of $N(X \times I) \times N G_{\mathbf{N}}$ generated by the simplices $\{((x, t), \operatorname{car}(H(x, t)))\}$.

(b) Let $B=A / \sim$, where $\sim$ is the equivalence relation:

(3.2) Given $((x, t), \sigma) \&((x, s), \sigma) \in A_{n}$, for any $n \geqslant 0,((x, t), \sigma) \sim((x, s), \sigma)$ iff $\left(\left(x, t^{\prime}\right), \sigma\right) \in A_{n}$ for every $t^{\prime} \in \overline{t s}(\overline{t s}$ is the interval between $t$ and $s)$.

(c) Let $C$ be the top-cat: $\operatorname{Ob}(C)=B_{0}, \operatorname{Mor}(C)=B_{0} \times_{X} B_{0}$ with the obvious groupoid structure.

The fibre product $B_{0} \times_{X} B_{0}$ is performed (on both sides) over the 0-component of the simplicial map $B \rightarrow N X$, obtained from the diagram:

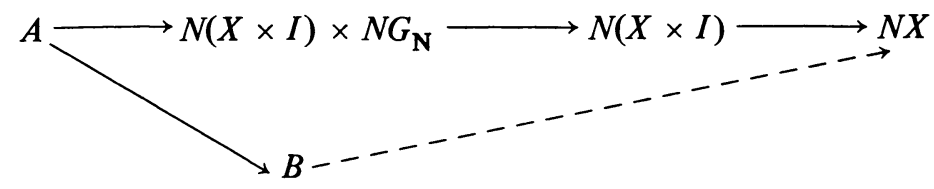

Note from the definition that there is a unique simplicial map $\beta: B \rightarrow N C$ for which $\beta_{0}=\mathrm{id}_{B_{0}}$.

(ii) Construction of the $j$ 's.

(3.3) Observe that $A$ is the nerve of $(X \times I)_{H}$ as constructed in (i) of Proposition 1.

Then, (2.4) together with the obvious functoriality of the construction gives us inclusions $N X_{p_{k}} \rightarrow A$ (where, as from now on, $k=0,1$ ). Now, the composite simplicial maps $N X_{p_{k}} \rightarrow A \rightarrow B \stackrel{\beta}{\rightarrow} N C$ are the nerves of functors $j_{k}: X_{p_{k}} \rightarrow C$.

Observing that $\mathrm{Ob}\left(X_{p}\right)=\mathrm{Ob}\left(X_{p_{0}}\right) \amalg O b\left(X_{p_{1}}\right)$, the object map of $j: X_{p} \rightarrow C$ is forced upon us. And on morphisms simply put $\operatorname{Mor}(j)=(\mathrm{Ob}(j) \circ S, \mathrm{Ob}(j) \circ T)$.

(iii) Construction of $F$. Here, we have to look at the construction of $C$ more closely. From the inclusion $A \subset N(X \times I) \times N G_{\mathrm{N}}$ we obtain projection simplicial maps:

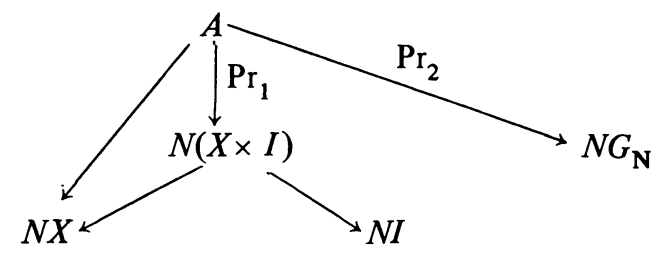

(3.4) $\operatorname{Pr}_{1}$ restricted to any component of $A_{n}$ (for any $n \geqslant 0$ ) is a homeomorphism onto an open set of $X \times I=N(X \times I)_{n}$. 
This follows from (3.3) and (2.4). (Moreover, $A_{0}$ can be considered as an open cover of $X \times I$, and $A_{n}$ consists of the $n$-fold intersections of the "components" of $A_{0}$.)

(3.5) Given $a$ and $b$ in $A_{n}$, then $a \sim b$ (in the sense of (3.2)) iff for some $x \in X, a$ and $b$ lie in the same component of $\left(A_{x}\right)_{n}$, where $A_{x}$ denotes the simplicial fibre in $A$ over $x$.

One implication is obvious. For the other, suppose $a$ and $b$ lie in the same component of $\left(A_{x}\right)_{n}$. Note from (3.4) that every component of $\left(A_{x}\right)_{n}$ is homeomorphic to an open interval in $I$.

Since $H$ is a linear-homotopy, $H_{x}: I \rightarrow B G$ factors through $B G^{\delta} \rightarrow B G$. Then, the projection $\operatorname{Pr}_{2} \mid A_{x}: A_{x} \rightarrow N G_{\mathrm{N}}$ factors through $N G_{\mathrm{N}}^{\delta} \rightarrow N G_{\mathrm{N}}$; thus, $\operatorname{Pr}_{2}$ is constant on every component of $\left(A_{x}\right)_{n}$. From here, (3.5) easily follows.

(3.6) The projection $B \rightarrow N X$ is locally a homeomorphism (i.e. every point of $B_{n}$ has an open neighbourhood homeomorphic to an open set of $X$ ).

(3.4) and (3.5) reduce this to an easy exercise in point set topology. Namely, $U / \sim$ has the required property with $U$ an open subset of $X \times I$ and $(x, t) \sim(x, s)$ iff $\left(x, t^{\prime}\right) \in U$ for all $t^{\prime} \in \overline{t s}$.

Let $B_{x}$ be the simplicial fibre in $B$ over $x \in X$. The final step is to prove

(3.7) $B_{x}$ is a contractible simplicial set (i.e., $B_{x}$ is discrete as subsimplicial space of $B$, and $\left|B_{x}\right|$ is contractible).

(3.5) implies that $\left(B_{x}\right)_{n}$ has a point for every connected component of $\left(A_{x}\right)_{n}$ (one could write $\left.B_{x}=\Pi_{0}\left(A_{x}\right)\right)$, and (3.6) implies that every point of $\left(B_{x}\right)_{n}$ is open. Thus, $B_{x}$ is discrete.

Before giving an explicit description of $B_{x}$, let us see how its contractibility gives a natural definition for $F$ :

There is an obvious simplicial map $F^{\prime}: B \rightarrow N G$ (recall part (i)). Thus, $F$ is already defined on objects $\left(\mathrm{Ob}(C)=B_{0}\right)$. Now, given $(a, b) \in \operatorname{Mor}(C)$, we have that $a \& b \in\left(B_{x}\right)_{0}$ for some $x \in X$. Then, since $B_{x}$ is connected, we can find a simplicial path in $B_{x}$ :

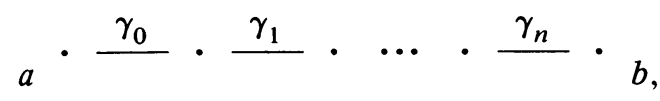

where " $\ldots$ " can be " $\leftarrow$ " or " $\rightarrow$ ". Then define

$$
F(a, b)=F_{1}^{\prime}\left(\gamma_{n}\right)^{\varepsilon_{n}} \circ \cdots \circ F_{1}^{\prime}\left(\gamma_{0}\right)^{\varepsilon_{0}}
$$

where $\varepsilon_{i}$ is 1 or -1 according to the orientation of $\gamma_{i}$. Since $B_{x}$ is simply-connected, any two paths as above are simplicially homotopic. And, using $F_{2}^{\prime}$, one easily sees that simplicial moves do not affect the outcome of (3.8). Thus, $F$ is well defined. Finally, the continuity of $F$ follows from (3.6) (any simplicial path as above can be extended over a small neighborhood of $x$ ). 
To describe $B_{x}$ explicitly, consider the map $\tau=\left|\pi_{1}\right| \circ H_{x}: I \rightarrow \Delta^{\infty}$. Then $A_{x}$ is the nerve of $I_{\tau}$ (recall (2.2), (2.4) and (3.3)).

Let $\left\{U_{\alpha}\right\}_{\alpha \in \Sigma}$ be the open cover of $I$ consisting of the connected components of $\left(A_{x}\right)_{0}=\operatorname{Ob}\left(I_{\tau}\right)$. We have a function $\Psi: \Sigma \rightarrow \mathbf{N}$, for which $\left\{U_{\alpha} \mid \alpha \in \Psi^{-1}(i)\right\}$ is the set of components of the open subset of $I:\{t \in I \mid i \in \operatorname{Image}(\operatorname{car}(\tau(t)))\}$.

Now $B_{x}$ can be described as the simplicial set with a vertex $u_{\alpha}$ for each $\alpha \in \Sigma$ and an $n$-simplex $\left\langle u_{\alpha_{0}}, \ldots, u_{\alpha_{n}}\right\rangle$ whenever $\bigcap_{i=0}^{n} U_{\alpha_{i}} \neq \varnothing$ and $\Psi\left(\alpha_{0}\right) \leqslant \Psi\left(\alpha_{1}\right) \leqslant \cdots \leqslant$ $\Psi\left(\alpha_{n}\right)$. To give an explicit contraction of $B_{x}$, note that we can find a finite sequence $\alpha^{0}, \alpha^{1}, \ldots, \alpha^{k}$, such that for each $\alpha \in \Sigma$, we either have (a) $U_{\alpha} \subset U_{\alpha^{i}}$ for a unique $i$; or (b) $U_{\alpha} \subset U_{\alpha^{i-1}} \cap U_{\alpha^{i}}$ and $U_{\alpha} \cap U_{\alpha^{j}}=\varnothing$ for $j \neq i-1$, i. (Put $\operatorname{lub}\left(U_{\alpha^{0}}\right)=$ $\max \left\{\operatorname{lub}\left(U_{\alpha}\right) \mid 0 \in U_{\alpha}\right\}$, and proceed by induction using that each $t \in I$ is in exactly $\operatorname{dim}(\operatorname{car}(\tau(t)))$ of the $U_{\alpha}$ 's.) Then, retract $B_{x}$ to the path $\left\langle u_{\alpha^{0}}, u_{\alpha^{1}}\right\rangle, \ldots,\left\langle u_{\alpha^{k-1}}, u_{\alpha^{k}}\right\rangle$ by sending $u_{\alpha}$ to $u_{\alpha^{i}}$ if (a) holds, or to the barycenter of $\left\langle u_{\alpha^{i-1}}, u_{\alpha^{i}}\right\rangle$ if (b) holds, and extending linearly. The path is contractible. Hence, we have completed the proof of the theorem.

Appendix. The Product Theorem. We give an elementary proof of Milnor's Product Theorem for simplicial spaces. Different versions of it are well known and widely used, but the following one (whose proof follows essentially Milnor's original one [M]) does not appear in the literature. The right setting to work in is that of bisimplicial spaces.

Given a bisimplicial space $E$ (i.e., a functor $E: \Delta^{\mathrm{op}} \times \Delta^{\mathrm{op}} \rightarrow$ Top, where $\Delta$ is the category of the ordered sets $\mathbf{n}=\{0,1, \ldots, n\})$, one has three simplicial spaces naturally associated to it: the diagonal $E^{\Delta}$; the vertical realization $E^{v}$, obtained by realizing the columns of $E$; and the horizontal realization $E^{h}$.

THEOREM 1. For any bisimplicial space $E:\left|E^{\Delta}\right|=\left|E^{v}\right|=\left|E^{h}\right|$.

(Here, equality means canonical homeomorphism, realization is the usual one ([S] or (1.3)), and everything takes place in the classic category of topological spaces.)

Proof. Consider the diagram

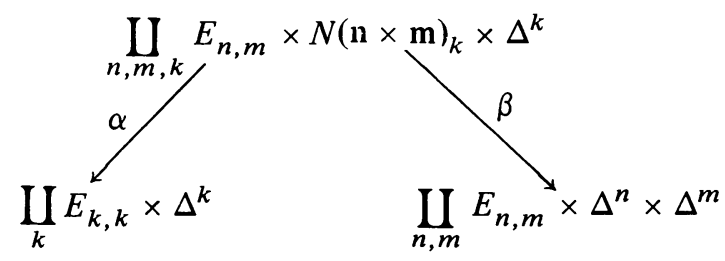

To define $\alpha$ and $\beta$, recall that a $k$-simplex of the nerve of $\mathbf{n} \times \mathbf{m}$ is a functor $\sigma$ : $\mathbf{k} \rightarrow \mathbf{n} \times \mathbf{m}$; denote its projections by $\sigma_{1}: \mathbf{k} \rightarrow \mathbf{n}$ and $\sigma_{2}: \mathbf{k} \rightarrow \mathbf{m}$. Then put

$$
\alpha(e, \sigma, t)=\left(\left(\sigma_{1}, \sigma_{2}\right)^{*}(e), t\right), \quad \beta(e, \sigma, t)=\left(e,\left|\sigma_{1}\right|(t),\left|\sigma_{2}\right|(t)\right) .
$$


(A.2) Let $\|E\|$ be the quotient of $\amalg_{n m} E_{n, m} \times \Delta^{n} \times \Delta^{m}$ under the relation $\left(e, \mathbf{f}_{*} t_{1}, \mathbf{g}_{*} t_{2}\right) \sim\left((\mathbf{f}, \mathbf{g})^{*} e, t_{1}, t_{2}\right)$ (for all obvious choices).

Clearly, the fibres of $\beta$ composed with the quotient map to $\|E\|$ correspond to the fibres of $\alpha$ followed by the quotient to $\left|E^{\Delta}\right|$. Thus, to conclude that $\left|E^{\Delta}\right|=\|E\|$ we show that $\alpha$ and $\beta$ are quotient maps:

$\alpha$ is a quotient map for it has a section $\delta(e, t)=\left(e,\left(\mathrm{id}_{\mathrm{k}}, \mathrm{id}_{\mathrm{k}}\right), t\right)$ (which includes the obvious diagonal mappings).

With $\beta$, one runs into the classic nuisance: "the product of quotients is not necessarily a quotient". However, we get by with the following elementary lemma, whose proof follows literally that of Hu's book [HU, Lemma (IV, 6.1), p. 143].

Definition. A quotient map $q: X \rightarrow Y$ is locally compact if for every pointed neighborhood $(y, V)$ in $Y$ there exists an open set $U$ of $X$ with compact closure contained in $q^{-1}(V)$, and such that $q(U)$ is an open neighborhood of $y$.

LEMMA. If one of the quotient maps $p, q$ is locally compact, then $p \times q$ is a quotient map.

The Lemma applies to the components of $\beta$ : "fix $n, m$ "; the hypothesis being fulfilled by the second factors. Hence, $\beta$ is a quotient map and $\left|E^{\Delta}\right|=\|E\|$.

The theorem now follows by proving that $\|E\|=\left|E^{h}\right|$ (and symmetrically $\|E\|=$ $\left.\left|E^{v}\right|\right)$. But this is obvious from the fact that the composition

$$
\coprod_{n, m} E_{n, m} \times \Delta^{n} \times \Delta^{m} \rightarrow \coprod_{m}\left|\left\{\mathbf{n} \mapsto E_{n, m}\right\}\right| \times \Delta^{m} \rightarrow\left|E^{h}\right|
$$

is a quotient map (apply the Lemma $-\Delta^{m}$ is compact).

As a corollary to the proof we obtain a sharp generalization of Milnor's Product Theorem [M].

THEOREM 2. Let $A$ and $B$ be simplicial spaces. If the quotient map $\amalg_{n} B_{n} \times \Delta^{n} \rightarrow|B|$ is locally compact, then $|A \times B|=|A| \times|B|$.

Proof. Consider the bisimplicial space $\left\{E_{n, m}=A_{n} \times B_{m}\right\}$. The Lemma implies that $|A| \times|B|=\|E\|$ (recall (A.2)). And, on the other hand, $E^{\Delta}=A \times B$.

\section{REFERENCES}

[B] J. Bracho, Strong classification of Haefliger Structures; some geometry of BG, Proc. Adem's Internat. Topology Sympos., Oaxtepec, Amer. Math. Soc., Providence, R. I., 1981.

[H] A. Haefliger, Homotopy and integrability, Lecture Notes in Math., vol. 197, Springer-Verlag, Berlin and New York, 1971, pp. 133-163.

[Hu] Sze-Tsen Hu, Elements of general topology, 3rd ed., Holden-Day, San Francisco, Calif., 1969.

[M] J. Milnor, The realization of a semi-simplicial complex, Ann. of Math. (2) 63 (1957), 272-284.

[S] G. Segal, Classifying spaces and spectral sequences, Inst. Hautes Études Sci. Publ. Math. 34 (1968), $105-112$.

InSTITUto de MATEMÁticas, UNAM, CD. UNIVERSITARIA, CIRCuíto EXTERIOR, MEXico D. F., 04510 